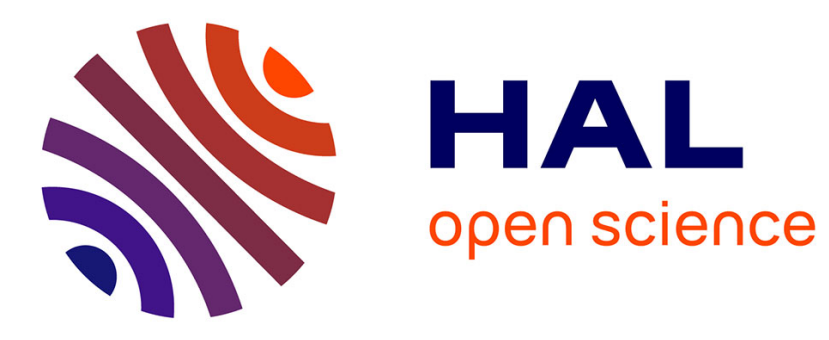

\title{
Magnetic Field Dependence of Spatial Frequency Encoding NMR
}

Bertrand Plainchont, Jonathan Farjon, Nicolas Giraud

\section{To cite this version:}

Bertrand Plainchont, Jonathan Farjon, Nicolas Giraud. Magnetic Field Dependence of Spatial Frequency Encoding NMR. encyclopedia of magnetic resonance, 2016, 10.1002/9780470034590.emrstm1528 . hal-03130775

\section{HAL Id: hal-03130775 \\ https://hal.science/hal-03130775}

Submitted on 3 Feb 2021

HAL is a multi-disciplinary open access archive for the deposit and dissemination of scientific research documents, whether they are published or not. The documents may come from teaching and research institutions in France or abroad, or from public or private research centers.
L'archive ouverte pluridisciplinaire HAL, est destinée au dépôt et à la diffusion de documents scientifiques de niveau recherche, publiés ou non, émanant des établissements d'enseignement et de recherche français ou étrangers, des laboratoires publics ou privés. 


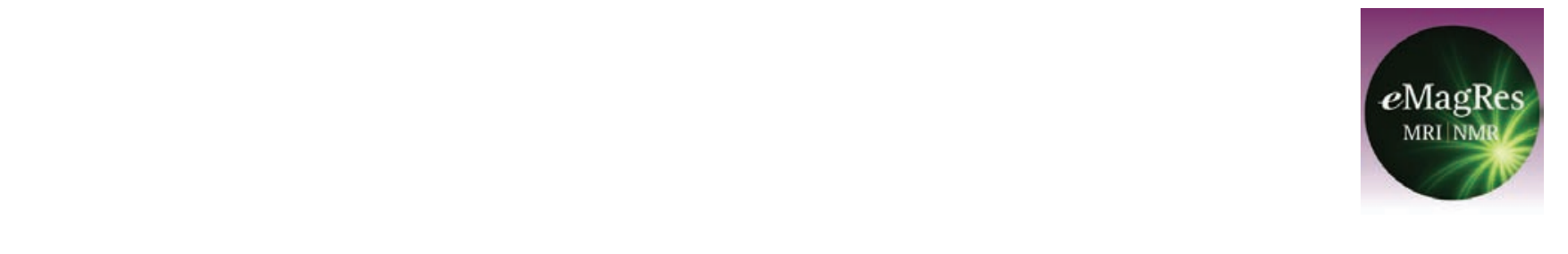

\title{
Magnetic Field Dependence of Spatial Frequency Encoding NMR
}

\author{
Bertrand Plainchont, Jonathan Farjon \&t Nicolas Giraud \\ Université Paris-Sud, Orsay Cedex, France
}

The influence of the strength of the static magnetic field on pulse sequences based on the concept of spatial frequency encoding is addressed. The evolution of the slice selection process occurring during encoded excitation and refocusing pulses, as well as pure shift and J-edited refocusing blocks, is studied both theoretically and experimentally on a model $A B X{ }^{1} \mathrm{H}$ spin system. It is shown that carrying out gradient-encoded pulse sequences at higher field enhances the quality of the spin dynamics that is locally triggered in each encoded slice and results in spectra of better resolution and sensitivity.

Keywords: high-resolution NMR, spatial frequency encoding, selective pulses, magnetic field gradient, magnetic field, sensitivity, resolution, pure shift, J-edited

How to cite this article:

eMagRes, 2016, Vol 5: 1-6. DOI 10.1002/9780470034590.emrstm1528

\section{Introduction}

Spatial frequency encoding (SFE) NMR is at the root of a series of methodological developments that have paved the way to the acquisition of ${ }^{1} \mathrm{H}$ 1-D and 2-D experiments with unprecedented high resolution. This method is based on the general concept of gradient-encoded pulses, which have been extensively used for a wide range of applications in NMR spectroscopy, depending on whether the time evolution of a spin system (single scan or ultrafast $\mathrm{NMR}^{1}$ ), the local spin composition (imaging ${ }^{2}$, diffusion ${ }^{3}$ ), or more generally, any spin dynamics process need to be edited throughout the sample. ${ }^{4}$ In the latter case, a SFE is needed to control locally the selectivity of the excitation, the decoupling, or the refocusing of individual spin nuclei from each site of the analyzed molecule, in contrast to the spatial encoding of a time domain that is performed in ultrafast NMR. ${ }^{5}$ In this spirit, Zangger and Sterk had first shown that broadband homonuclear decoupling sequences based on a SFE can be performed efficiently by implementing a pure shift (also known as $\delta$-resolved) refocusing block. ${ }^{6}$ More recently, we have shown that the selective refocusing of homonuclear scalar or residual dipolar couplings can also be encoded along the sample, according to the resonance frequency of each coupling partner. ${ }^{7,8}$ We have also demonstrated that it is possible to combine pure shift and J-edited spin evolutions into fully tailored experiments and produce ultrahigh-resolution correlation spectra with exactly the desired analytical content. ${ }^{9,10}$ The high flexibility of SFE methods has also opened the way to an implementation of both pure shift and J-edited blocks into real-time acquisition schemes. ${ }^{11-13}$

In a recent paper, we have reported the acquisition of highresolution gradient-encoded correlation experiments on an oligomeric saccharide sample showing a highly crowded ${ }^{1} \mathrm{H}$ spectrum. ${ }^{14}$ We have experimentally shown that operating at higher magnetic field leads to a better efficiency of pure shift as well as J-edited refocusing blocks based on a SFE, leading to noticeable resolution enhancements on this spectroscopically challenging sample. In the present work, we study the magnetic field dependence of SFE NMR on a model ABX ${ }^{1} \mathrm{H}$ spin system. Notably, we describe the evolution of spatial resolution and sensitivity when going to higher field. We use the theoretical formalism that we have introduced recently ${ }^{15}$ to simulate the spatial properties of the investigated irradiation schemes throughout the sample during a slice selection process.

\section{Magnetic Field Dependence of the Slice Selection Process}

\section{Excitation Step}

SFE results from the simultaneous application of a selective pulse and a pulsed magnetic field gradient. The basic idea underlying this irradiation scheme is that proton spins with different resonance frequencies are selectively irradiated in different 'slices' of the sample. The key features of this process can be understood through the basic single gradient-encoded excitation pulse sequence depicted in Figure 1(a). In the following, 3-hydroxy-4,4,4-trichlorobutyric $\beta$-lactone 1 (Figure 1b) is chosen as a model system to study the magnetic field dependence of the SFE. During a gradient-encoded pulse, the strength of the total magnetic field depends on the position $z$ in the sample due to the application of a pulsed field gradient (Figure 1c). As a result, each proton site $H^{A}, H^{B}$, and $H^{X}$ will interact with the encoded selective pulse only around the position $z_{A}$ (respectively, $z_{B}$ and $z_{X}$ ) that satisfies the relation:

$$
v^{A, B, X}=\frac{\gamma_{H}}{2 \pi} \cdot\left\{B_{0}+G_{z} \cdot z_{A, B, X}\right\}\left\{1-\sigma_{A, B, X}\right\}
$$




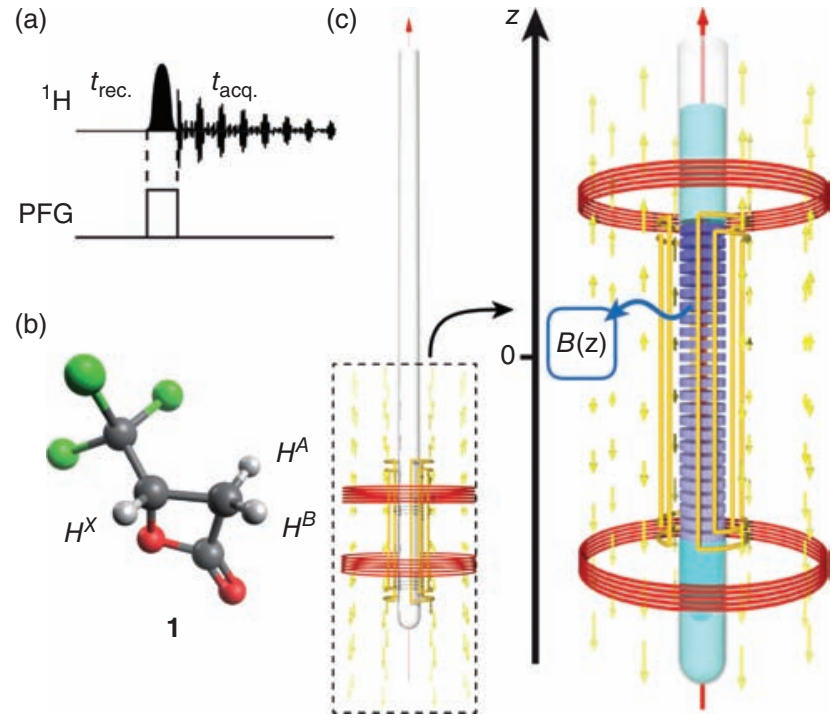

Figure 1. (a) The single gradient-encoded pulse sequence. The delays $t_{\text {rec }}$ and $t_{\text {acq. }}$ are the recovery and the acquisition times, respectively. The black ellipsoidal shape on the proton channel $\left({ }^{1} \mathrm{H}\right)$ corresponds to a shaped excitation pulse. The white rectangular bar on the pulsed field gradient (PFG) channel refers to the application of a rectangular-shaped $z$ field gradient. (b) The structure of the model ${ }^{1} \mathrm{H} A B X$ spin system 1. (c) Schematic representation of the spatial frequency encoding performed during the single gradient-encoded shaped pulse experiment shown in (a). The receiver coil is represented in orange in order to materialize the total height of the sample that is detected. The $z$ gradient coils are pictured in red. Yellow arrows symbolize the magnetic field gradient

where $v^{A}$ and $\sigma_{A}$ (respectively, $v^{B}$ and $\sigma_{B}$, and $v^{X}$ and $\sigma_{X}$ ) are the resonance frequency and the shielding of $H^{A}$ (respectively, $H^{B}$ and $\left.H^{X}\right), B_{0}$ the external static magnetic field generated by the superconducting magnet, $\gamma_{H}$ the gyromagnetic ratio of the proton, and $G_{z}$ the strength of the magnetic field gradient taken here along the $z$-axis.

Figure 2 shows the spatial distribution of the magnetization corresponding to the coherences $\mathrm{A}_{+} \mathrm{B}_{\alpha} \mathrm{X}_{\alpha}$ and $\mathrm{A}_{\alpha} \mathrm{B}_{\alpha} \mathrm{X}_{+}$of $H^{A}$ and $H^{X}$ calculated at the end of a gradient-encoded excitation step, at $14.1 \mathrm{~T}\left(v^{H}=600 \mathrm{MHz}\right)$ and $23.5 \mathrm{~T}\left(v^{H}=1 \mathrm{GHz}\right)$. For each magnetic field strength $B_{0}$, the strength of the encoding gradient $G_{B_{0}}$ is set so that the same range of chemical shifts, corresponding to the spectrum of the analyzed compound, is encoded along the virtual NMR sample. A larger spectral width $\gamma G_{B_{0}} l$, where $\gamma$ is the gyromagnetic ratio and $l$ the length of the sample that is detected by the receiver coil, is thus encoded along the sample at higher field. Moreover, the same E-Burp2 pulse of identical duration is used at both fields, which ensures the same spectral bandwidth $\Omega_{\mathrm{B}}$. For $H^{X}$ first, the region around $v^{X}$ within which the corresponding transverse magnetization is created is found to be thinner at $23.5 \mathrm{~T}$ than at $14.1 \mathrm{~T}$, which is coherent with the fact that at first approximation the fraction of the sample that is selected by the gradient-encoded pulse is proportional to $\Omega_{\mathrm{B}} /\left(\gamma G_{B_{0}} l\right) .{ }^{16}$ We also remark that the shape of the phase distribution within the region encoded around $v^{X}$ is comparable at both fields, although being thinner at 23.5 T. Moreover for $H^{A}$, it is apparent that at $14.1 \mathrm{~T}$, a selectivity issue arises as transverse magnetization corresponding (a) $14.1 \mathrm{~T} \quad \mathrm{~A}_{\alpha} \mathrm{B}_{\alpha} \mathrm{X}_{+}$

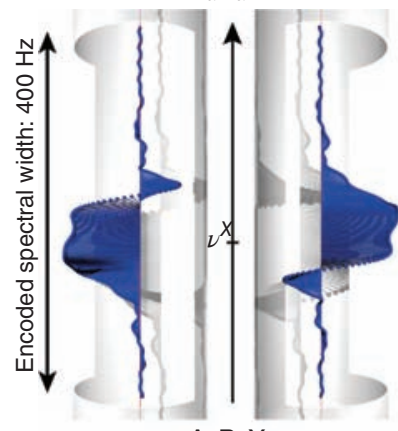
$\mathrm{A}_{+} \mathrm{B}_{\alpha} \mathrm{X}_{\alpha}$

(b) $23.5 \mathrm{~T} \quad \mathrm{~A}_{a} \mathrm{~B}$

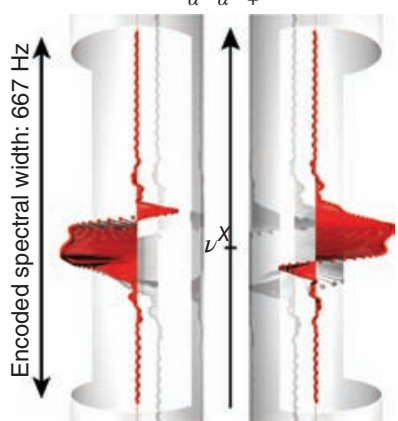

$\mathrm{A}_{+} \mathrm{B}_{\alpha} \mathrm{X}_{\alpha}$
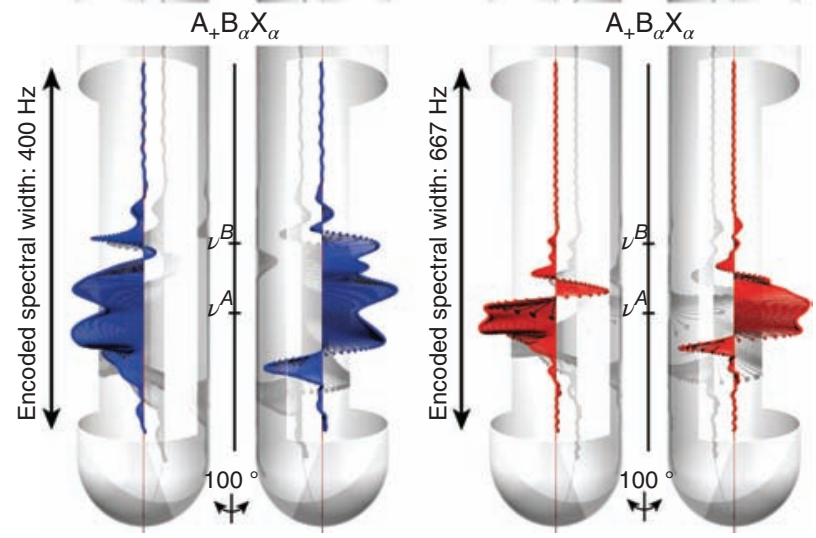

Figure 2. The spatial variation of the magnetization calculated at (a) 14.1 and (b) $23.5 \mathrm{~T}$ at the end of a gradient-encoded excitation pulse is represented with 2 viewing angles inside the NMR tube, for (top) $\mathrm{A}_{\alpha} \mathrm{B}_{\alpha} \mathrm{X}_{+}$and (bottom) $\mathrm{A}_{+} \mathrm{B}_{\alpha} \mathrm{X}_{\alpha}$ coherences. An E-Burp2 pulse of duration $60 \mathrm{~ms}$ was used for the selective irradiation. The virtual sample was divided into 401 slices of equal height around each region of interest, to which a positiondependent magnetic field value was assigned

to the $\mathrm{A}_{+} \mathrm{B}_{\alpha} \mathrm{X}_{\alpha}$ term is observed not only around $v^{A}$ but also around $v^{B}$, which means that the gradient-encoded excitation pulse cannot select $H^{A}$ and $H^{B}$ in spatially resolved cross sections. For the simulation carried out at $23.5 \mathrm{~T}$, the thinner region that is observed around $v^{A}$, combined with the greater difference between the Larmor frequencies of $H^{A}$ and $H^{B}$, contributes to improve the selectivity of the excitation pulse. As a result, very few residual magnetization is detected around $v^{B}$, which indicates that at $23.5 \mathrm{~T}$ the selectivity of the gradientencoded pulse is good enough to excite $H^{A}$ and $H^{B}$ in distinct slices of the sample.

Furthermore, the ${ }^{1} \mathrm{H}$ spectra resulting from the SFE described above can be reconstructed by adding the NMR signals that are locally encoded for each observable coherence of the model spin system 1. In Figure 3, the spatial distribution of NMR spectra generated locally by the gradient-encoded excitation pulse is shown as a pseudo-2-D plot at 14.1 and 23.5 T. At 14.1 T, we note that $H^{A}$ and $H^{B}$ are encoded in overlapped cross sections, as it is highlighted by the amplitude profile displayed within the NMR tube. The overall spectrum that is the sum of these subspectra shows distorted lines, which arises directly from this spatial resolution issue. Again, it is apparent that operating at higher field allows for solving this problem, as at $23.5 \mathrm{~T}$ two cross sections are clearly distinguishable at
61

62 63

64 65

66 67 68 69 70 71 72 73 74 75 76 77 78 79 80 81 82 83 84 85 86 87 88 


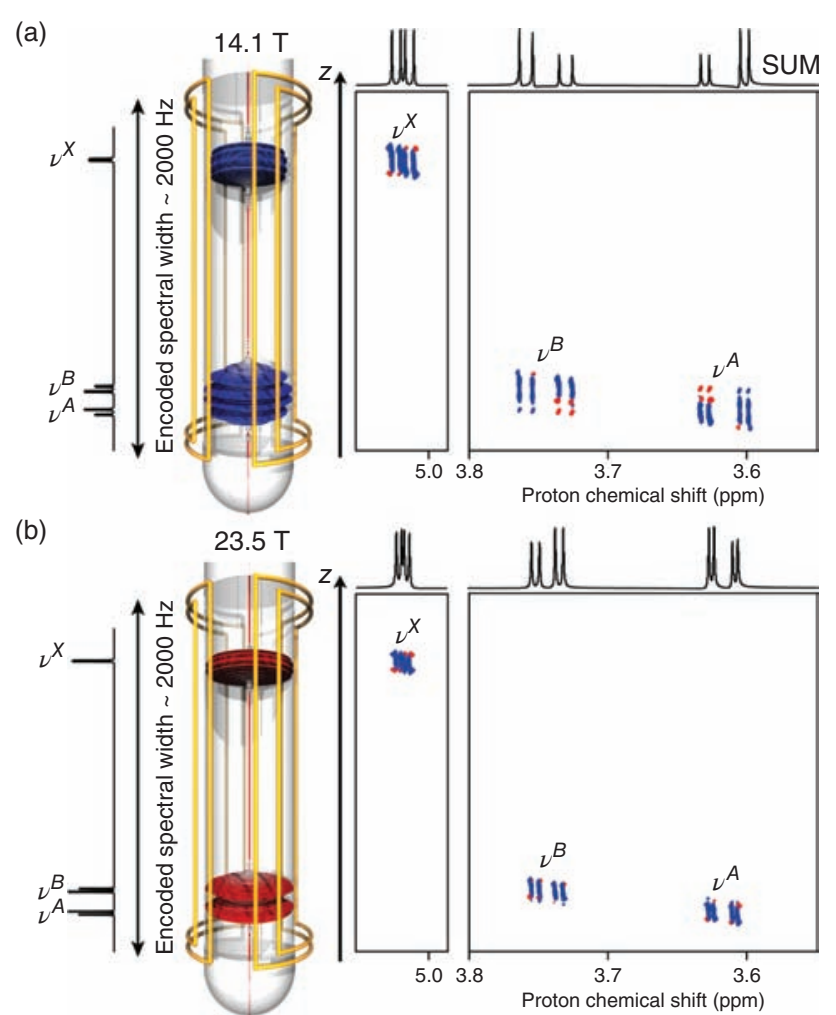

Figure 3. The amplitude profile of the NMR signal evaluated on the first point of each locally generated free induction decay is represented in cylindrical coordinates at $14.1 \mathrm{~T}$ (a) and $23.5 \mathrm{~T}$ (b). For each magnetic field, the spatial distribution of virtual proton spectra is reported in pseudo-2-D plots where the vertical axis refers to the position $z$ in the NMR sample. On these maps, blue and red contours are positive and negative, respectively

$v^{A}$ and $v^{B}$, respectively, leading to a ${ }^{1} \mathrm{H}$ spectrum showing the classical roof effect expected for a second-order spin system.

In conclusion for this part, the implementation of a SFE at higher magnetic field allows for improving the spatial resolution of the slice selection process, by selecting thinner cross sections throughout the sample.
Influence of the Shape of the Gradient-encoded Pulse

In a previous paper, we had shown that among the different shaped pulses designed for the purpose of selective irradiations, not all of them are suitable for undergoing a SFE. ${ }^{15}$ We had established that a key feature for yielding a proper slice selection process is the ability for a given shape to maintain the distribution of magnetization coherent along the encoded cross section. Figure 4 shows the evolution of the slice selection process simulated at 14.1 and $23.5 \mathrm{~T}$ for a series of excitation shaped pulses. Their duration was set so that they all have the same spectral bandwidth. For every shape, we observe a thinning of the encoded slices when going to higher field, as it can be monitored on the signals located at $v^{X}$. An improvement of the slice selection process is notably observed at higher field for E-Burp2 $\left(90^{\circ}\right)$, Esnob $\left(270^{\circ}\right)$, as well as Gaussian pulses calibrated for both $90^{\circ}$ and $270^{\circ}$, as it is illustrated by the presence at $23.5 \mathrm{~T}$ of two distinct slices at $v^{A}$ and $v^{B}$. We note that this was less predictable for the Gaussian $90^{\circ}$ pulse that had been previously shown to suffer from a problem of phase coherence upon gradient encoding. These results highlight the suitability of E-Burp2, Esnob, and Gaussian shapes for SFE NMR.

Influence of $B_{0}$ on a Gradient-encoded Refocusing Pulse

Gradient-encoded refocusing pulses are key actors of the spin dynamics that is locally triggered in each region of the sample, because they have been shown to improve greatly the spatial selectivity of the refocusing blocks within which they are implemented. In order to evaluate the influence of $B_{0}$ on this kind of selective pulse, we have simulated the action of a gradient-encoded Reburp $\pi$ pulse on transverse magnetization initially taken along the $y$-axis and composed of selected coherences of $H^{A}$ and $H^{X}$, at 14.1 and 23.5 T. In Figure 5, the spatial distribution of the resulting magnetization is shown for both magnetic fields. Similarly to what is observed for the excitation case, the cross sections within which the magnetization is properly refocused by the refocusing block are found to be thinner at higher field for both proton sites, whereas outside of these regions the initial transverse magnetization is fully dephased. We also note that the height of the cross section encoded at $v^{A}$ at $23.5 \mathrm{~T}$ allows for refocusing with a high

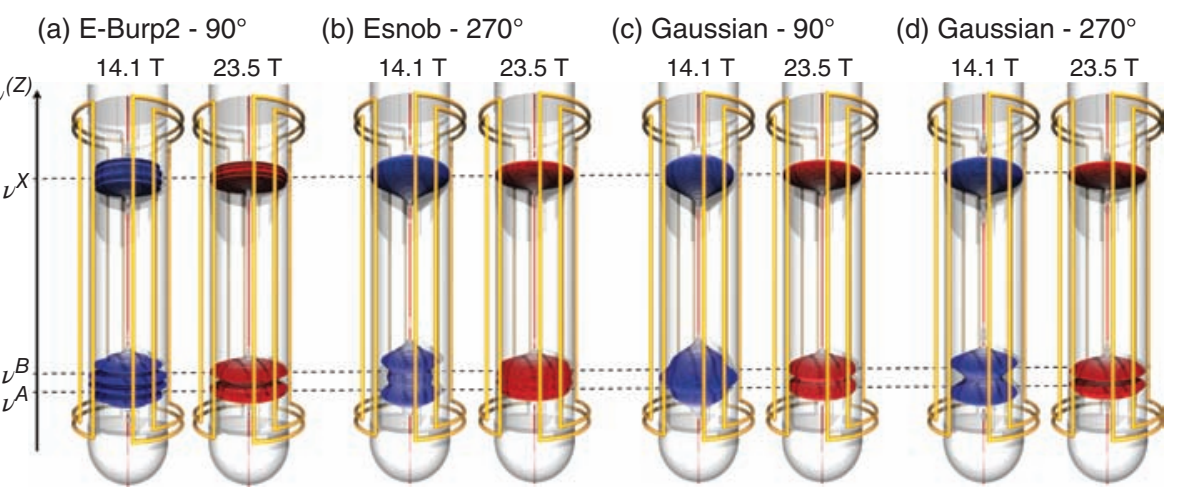

Figure 4. The evolution of the amplitude profile generated by a gradient-encoded excitation step is represented at 14.1 and $23.5 \mathrm{~T}$ for (a) an E-Burp2 $90^{\circ}$ pulse of $60 \mathrm{~ms}$, (b) an Esnob $270^{\circ}$ pulse of $20 \mathrm{~ms}$, (c) a Gaussian $90^{\circ}$ pulse of $20 \mathrm{~ms}$, and (d) a Gaussian $270^{\circ}$ pulse of $20 \mathrm{~ms}$ 


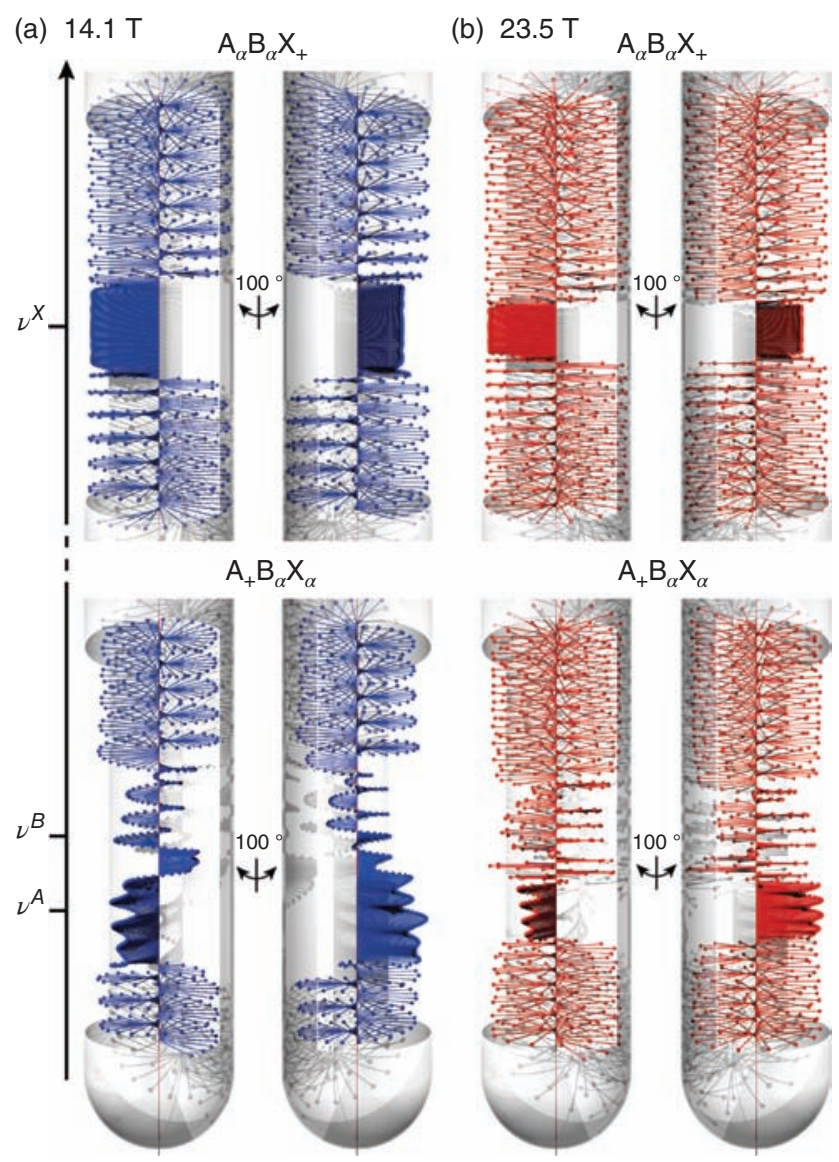

Figure 5. The spatial variation of the magnetization calculated at the end of gradient-encoded Reburp $180^{\circ}$ pulse of $60 \mathrm{~ms}$ at (a) $14.1 \mathrm{~T}$ and (b) 23.5 $\mathrm{T}$, for (top) $\mathrm{A}_{\alpha} \mathrm{B}_{\alpha} \mathrm{X}_{+}$and (bottom) $\mathrm{A}_{+} \mathrm{B}_{\alpha} \mathrm{X}_{\alpha}$ coherences

spatial selectivity the evolution of a coherence involving $H^{A}$ without affecting the spin state of $H^{B}$, which is a prerequisite for decoupling these two strongly coupled protons.

\section{From 1-D to 2-D Spatial Frequency Encoding NMR}

Following on from the above, the study of the magnetic field dependence of SFE NMR can be extended to the general case of a pulse sequence composed of several gradient-encoded pulses. As an illustration, we have acquired a 2-D $\omega_{1}$-homonuclear decoupled experiments ${ }^{17}$ at two different magnetic fields, on $10 \mathrm{mg}$ of $\mathbf{1}$ dissolved in $700 \mu \mathrm{l}$ of deuterated chloroform. We remind that this pulse sequence implements a pure shift block during the indirect evolution delay, which yields a 2-D phased spectrum with only diagonal peaks showing a singlet structure in the indirect domain, and a normal multiplet structure in the direct domain. For this experimental part, we have chosen to monitor the resolution enhancement that can be achieved in the pure shift dimension when going from $7.05 \mathrm{~T}\left(v^{H}=300\right.$ $\mathrm{MHz}$ ) to $14.1 \mathrm{~T}$. The resulting spectra are shown in Figure 6 together with the amplitude profiles that were calculated after the excitation step and at the end of the pure shift block of this pulse sequence. At $7.05 \mathrm{~T}$, we observe that neither the excitation step nor the pure shift block can achieve a slice selection process of sufficient spatial resolution for $H^{A}$ and $H^{B}$. On the resulting 2-D spectrum, it is apparent both protons were encoded in the same cross section, as it is illustrated by the shape of the distorted correlation appearing around $v^{A}$ and $v^{B}$. Conversely, at $14.1 \mathrm{~T}$, we note that the efficiency of the refocusing block allows for refining the slice selection process initially created during the excitation step, to a point where $H^{A}$ and $H^{B}$ can be selectively decoupled. The better spatial resolution that is reached at this field can be straightforwardly correlated with the high spectral resolution achieved at $v^{A}$ and $v^{B}$. The projection displayed along the indirect domain allows for observing the singlet structure of each correlation, which highlights the higher efficiency of the homonuclear decoupling sequence at $14.1 \mathrm{~T}$ than at 7.05 $\mathrm{T}$.

\section{Magnetic Field Dependence of Sensitivity}

One frequent reservation that is sometimes expressed about the relevance of SFE techniques regards their low sensitivity. To tackle this problem, it is important to discuss here whether acquiring spatially encoded spectra at higher field has an overall beneficial effect on sensitivity. In this section, we aim thus at quantifying the relationship between the sensitivity of a gradient-encoded experiment and $B_{0}$. We have studied, for eight different magnetic fields that are representative of the range of NMR spectrometers commercially available to date, the evolution of the sensitivity of a single gradient-encoded pulse experiment. The integration of the signal of $H^{X}$ and $H^{A}$ has been calculated for each magnetic field and normalized by the signal arising from the same semiselective excitation pulse without SFE (Figure 7). The resulting ratio $I_{\text {encoded }} / I_{\text {selective }}$ provides a direct quantification of the fraction of the sample within which $H^{X}$ and $H^{A}$ are encoded, while allowing to throw off the contribution of the intrinsic probehead sensitivity and the Boltzmann population of the observed spin. Again, we have set the gradient strength so that the spectral width that is encoded along the virtual sample matches exactly the proton spectrum of 1 . As expected, $I_{\text {encoded }} / I_{\text {selective }}$ values range from 4 to $18 \%$ and decrease with $B_{0}$, which reflects the thinning of the encoded cross section with the strength of the magnetic field. Furthermore, as $I_{\text {encoded }}$ is proportional to the spectral bandwidth of the encoded pulse, the resulting ratio should, to a first approximation, be proportional to $B_{0}{ }^{-1}$. Figure 7(a) shows the corresponding trend curve described by Pell and Keeler ${ }^{16}$ that we have adjusted to the ratio calculated at 23.5 $\mathrm{T}$ to account for the contribution of the shaped pulse to the slice selection process. For $H^{X}$, the evolution of the ratios is in agreement with this trend curve. For $H^{A}$, however, although the ratios obtained at higher field (14.1-23.5 T) follow the trend curve, those determined at lower field $(7.05,9.4$, and 11.7 T) are higher than expected, which is explained by the fact that the slice selection induced by the SFE could not be operated properly, resulting in an overlap of the two slices within which $H^{A}$ and $H^{B}$ are encoded, and hence the observed deviations. From this result, it is now possible to extrapolate the overall dependence of the sensitivity of a single gradient-encoded pulse experiment on $B_{0}$. For the homonuclear case, and if we
61

62 63 64 65 66 67 68 69 70 71 72 73 74 75 76 77 78 79 80 81 82 83 84 85 86 87 88 

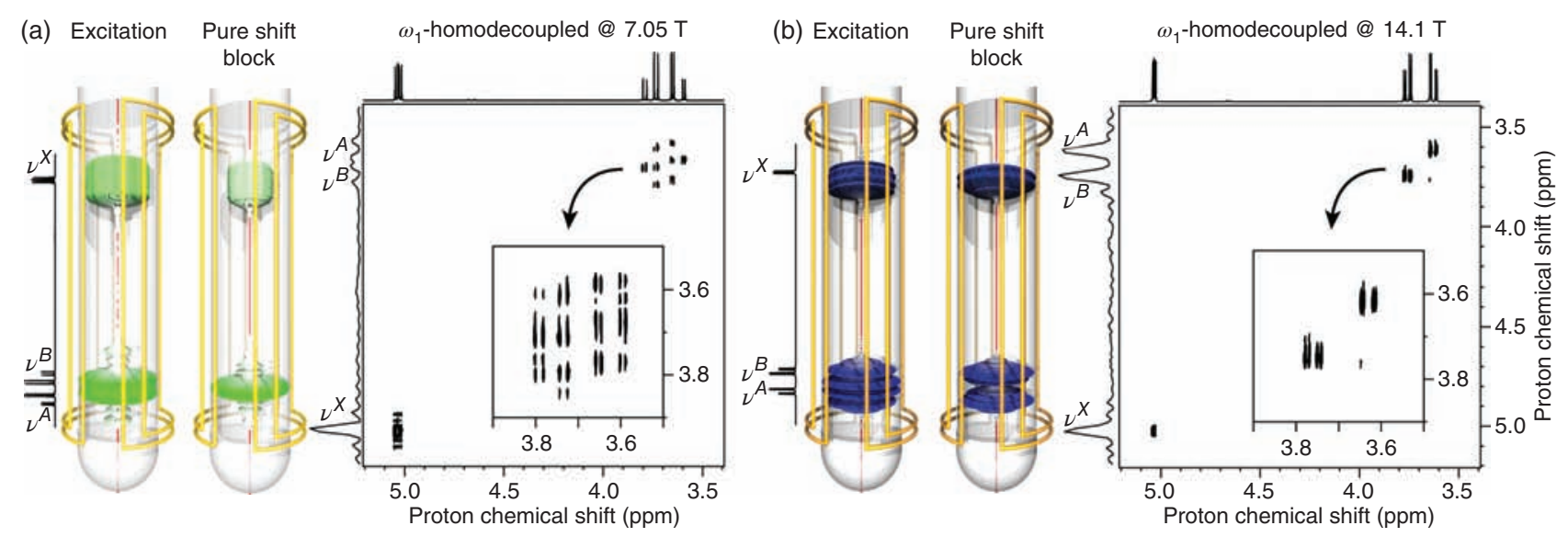

Figure 6. The amplitude profiles calculated after the gradient-encoded excitation pulse and at the end of the pure shift block of a 2-D ${ }^{1} \mathrm{H} \omega_{1}$-homonuclear decoupled experiment simulated on $\mathbf{1}$ at (a) 7.05 T and (b) 14.1 T. The corresponding experimental 2-D spectra acquired are also shown at both fields. E-Burp2 and Reburp pulses of $60 \mathrm{~ms}$ were used for the gradient-encoded excitation and the pure shift block, respectively. The strength of the gradient coil was set so as to encode along the sample a spectral width of 6.4 and $6.3 \mathrm{ppm}$ at 7.05 and $14.1 \mathrm{~T}$, respectively

assume comparable linewidths and probe performances, a rough estimate of the dependence of $S / N$ on $B_{0}$ is ${ }^{18}$

$$
S / N \propto n \cdot \gamma_{H}^{5 / 2} \cdot B_{0}^{3 / 2}
$$

where $n$ is the number of observed proton nuclei in the sample. The value of the integration that can be derived from $I_{\text {encoded }}$ to account for (i) the influence of the magnetic field on the Boltzmann population of the observed proton spins, (ii) the signal induced in the receiver coil at detection, and (iii) the noise has been calculated for different $B_{0} s$ (Figure $7 \mathrm{~b}$ ). On the one hand, we note that the overall sensitivity of the spatially frequency-encoded experiment increases with the strength of the magnetic field, despite the selection of thinner slices upon the gradient-encoded excitation step. On the other hand, for $H^{A}$, the NMR signal acquired at lower field is weaker than what could be expected from the ideal curve adjusted to the sensitivity measured at $23.5 \mathrm{~T}$, which underlines again the spatial resolution issue previously described at lower field. These results highlight the relevance of implementing spatially frequency-encoded pulse sequences at higher magnetic field, in so far as it contributes to enhance the three inextricably linked features that are the dispersion of the resonance frequencies (i.e., the spectral resolution), the spatial resolution of the slice selection process, and the sensitivity - although this latter point is modulated by the slice selection issue.

\section{Conclusion}

In this article, we have described the magnetic field dependence of NMR pulse sequences based on a SFE of the sample. We have highlighted the evolution of the key features that drive sensitivity and resolution in gradient-encoded pulse sequences, from the basic irradiation schemes, to state-of-the-art refocusing blocks that are nowadays routinely implemented in high-resolution experiments dedicated to the extraction of ${ }^{1} \mathrm{H}$ chemical shifts and/or homonuclear couplings. We have shown that operating at higher field leads to a better spatial resolution of the slice selection process, together with a moderate sensitivity enhancement of the resulting data. The combination of SFE techniques with high magnetic fields shades a new light on the trade-off that has classically to be found between sensitivity and resolution in SFE NMR. Indeed, the main limitation of high-resolution techniques based on this method regards the sensitivity penalty that has to be paid when protons with very close chemical shifts have to be decoupled, because it implies the use of highly selective pulses. ${ }^{19}$ We remark that operating at higher field amounts to increasing the chemical shift dispersion while keeping the same selectivity for the gradient-encoded pulses, which is a way to address this issue without sacrificing sensitivity. The implementation of SFE methods on high field spectrometers should thus be particularly suitable for the analysis of larger molecular systems showing overcrowded ${ }^{1} \mathrm{H}$ spectra.

\section{Acknowledgment}

This work was supported by the French Research Agency (ANR-2011-JS08-009-01).

\section{Biographical Sketches}

Bertrand Plainchont. b. 1985. MSc, Université de Strasbourg (France), 2009. PhD directed by J.-M. Nuzillard, Université de Reims Champagne-Ardenne (France), 2012. Postdoctoral research fellow at Université Paris-Sud, France. About 10 publications dealing with methodological developments for the simplification of complex liquid-state NMR spectra, computer-assisted structure elucidation and verification, and spin dynamics simulation.

Jonathan Farjon. b. in 1977, carried out a master in Chemistry at the Paris South University in 2000. PhD in chemistry, Paris South University 2003. Since 2009, research associate of the National Center for Scientific Research at the Paris South University. 23 publications on NMR in liquid and oriented media, methodology, and their applications in bio(in)organic chemistry. Actual interest is dedicated to highly resolved, sensitive, and quantitative techniques as well as fast NMR.

Nicolas Giraud. b. 1977. BSc, Ecole Normale Supérieure de Lyon (France) 2001. PhD, Chemistry, Université Claude Bernard Lyon I Lyon (France), 2005. Currently Reader in Chemistry at the Université 

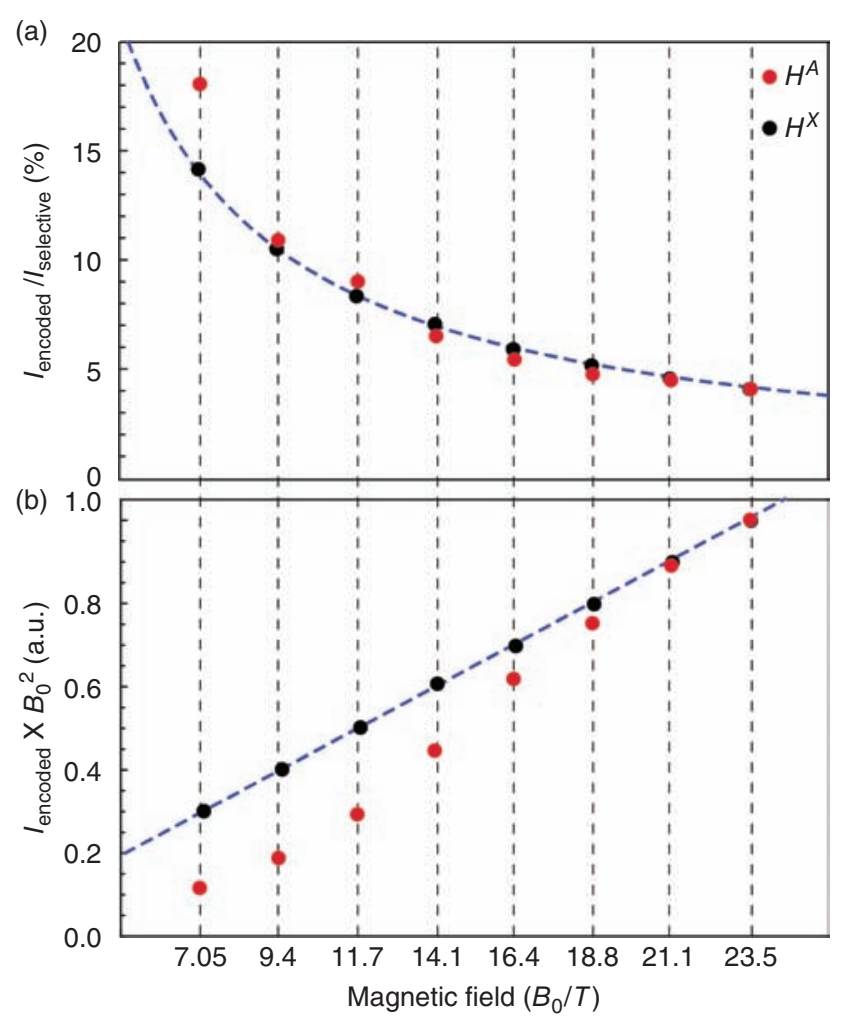

Figure 7. (a) The simulated sensitivity ratio between the integration of the signal resulting from a selective excitation with $\left(I_{\text {encoded }}\right)$ and without spatial frequency encoding $\left(I_{\text {selective }}\right)$ at eight different magnetic fields (from 7.05 to $23.5 \mathrm{~T}$ ), for $H^{A}$ and $H^{X}$. The trend curve corresponding to the ideal behavior of this integration ratio modeled by Keeler and Pell is plotted as a dashed line. (b) The evolution of the integration of the gradient-encoded signal ponderated by $B_{0}{ }^{2}$, as a function of $B_{0}$, for $H^{A}$ and $H^{X}$. The trend curve corresponding to the ideal evolution of this integration is plotted as a dashed line

Paris-Sud, France. About 25 publications on the development and the applications of liquid- and solid-state NMR techniques. Current research interest: development of new high-resolution NMR methods for the structural and dynamic analysis of complex molecular systems in chemistry and biology.

\section{Related Articles}

Modern NMR Pulse Sequences in Pharmaceutical R\&D; Pure Shift NMR Spectroscopy; Field Gradients and Their Application; Radiofrequency Pulses: Response of Nuclear Spins; Gradient Coil Systems; Radiofrequency Gradient Pulses; Selective Pulses; Shaped Pulses

\section{References}

61

1. L. Frydman, T. Scherf, and A. Lupulescu, Prog. Nucl. Magn. Reson. Spectrosc., 2002, 99, 15858. DOI: 10.1073/pnas.252644399.

2. M. K. Stehling, R. Turner, and P. Mansfield, Science, 1991, 254, 43.

3. C. S. Johnson Jr, Prog. Nucl. Magn. Reson. Spectrosc., 1999, 34, 203.

4. S. Berger, Prog. Nucl. Magn. Reson. Spectrosc., 1997, 30, 137. DOI: 10.1016/S0079-6565(97)00003-4.

5. A. Tal and L. Frydman, Prog. Nucl. Magn. Reson. Spectrosc., 2010, 57, 241.

6. K. Zangger and H. Sterk, J. Magn. Reson., 1997, 124, 486. DOI: 10.1006/jmre.1996.1063.

7. N. Giraud, L. Beguin, J. Courtieu, and D. Merlet, Angew. Chem. Int. Ed., 2010 49, 3481. DOI: 10.1002/anie.200907103.

8. D. Merlet, L. Beguin, J. Courtieu, and N. Giraud, J. Magn. Reson., 2011, 209, 315. DOI: 10.1016/j.jmr.2011.01.030.

9. N. Giraud, D. Pitoux, J. M. Ouvrard, and D. Merlet, Chem. Eur. J., 2013, 19, 12221. DOI: $10.1002 /$ chem.201302005.

10. D. Pitoux, B. Plainchont, D. Merlet, Z. Hu, D. Bonnaffé, J. Farjon, and N. Giraud, Chem. Eur. J., 2015, 21, 9044. DOI: 10.1002/chem.201501182.

11. N. H. Meyer and K. Zangger, Angew. Chem. Int. Ed., 2013, 52, 7143. DOI: 10.1002/anie.201300129.

12. N. Gubensak, W. M. F. Fabian, and K. Zangger, Chem. Commun., 2014, 50 , 12254. DOI: 10.1039/C4CC05892E.

13. N. Lokesh, S. R. Chaudhari, and N. Suryaprakash, Chem. Commun., 2014, 50 15597. DOI: 10.1039/C4CC06772J.

14. D. Pitoux, Z. Hu, B. Plainchont, D. Merlet, J. Farjon, D. Bonnaffé, and N. Giraud, Magn. Reson. Chem., 2015, 53, 836. DOI: 10.1002/mrc.4281.

15. B. Plainchont, D. Pitoux, G. Hamdoun, J. M. Ouvrard, D. Merlet, J. Farjon, and N. Giraud, Submitted, 2016

16. A. J. Pell and J. Keeler, J. Magn. Reson., 2007, 189, 293. DOI: 10.1016/j.jmr. 2007.09.002.

17. N. Giraud, M. Joos, J. Courtieu, and D. Merlet, Magn. Reson. Chem., 2009, 47 300. DOI: $10.1002 / \mathrm{mrc} .2387$.

18. R. R. Ernst, G. Bodenhausen, and A. Wokaun, Principles of Nuclear Magnetic Resonance in One and Two Dimensions, 2nd edn, Clarendon Press: Oxford, 1987.

19. M. Foroozandeh, R. W. Adams, M. Nilsson, and G. A. Morris, J. Am. Chem. Soc, 2014. DOI: $10.1021 /$ ja507201t.
62

63

64

65

66

67

68

69

70

71

72

73

74

75

76

77

78

79

80

81

82

83

84

85

86

87

88

89 
QUERIES TO BE ANSWERED BY AUTHOR

IMPORTANT NOTE: Please mark your corrections and answers to these queries directly onto the proof at the relevant place. 64 DO NOT mark your corrections on this query sheet.

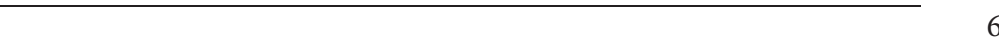

$\begin{array}{lr}\text { Queries: } & 68\end{array}$

$\begin{array}{lr}\text { AQ1. Please provide complete reference details for Ref. 15. } & 70 \\ \end{array}$

72

73

74

75

76

77

78

79

80

81

82

83

84

85

86

87

88
89

89
90

91

92

93

94

95

96

97

98

99

100

101

102

103

104

105

106

107

108

109

110

111

112

113

114

115

116

117

118

119 